\title{
Movement Quality Parameters During Gait Assessed by a Single Accelerometer in Subjects with Osteoarthritis and Following Total Joint Arthroplasty
}

Jill Emmerzaal ( $\square$ jill.emmerzaal@kuleuven.be )

KU Leuven https://orcid.org/0000-0002-9218-7604

Kristoff Corten

Ziekenhuis Oost-Limburg - Campus Sint Jan

Rob van der Straaten

UHasselt: Universiteit Hasselt

Liesbet De Baets

Vrije Universiteit Brussel

Sam Van Rossom

KU Leuven: Katholieke Universiteit Leuven

Annick Timmermans

UHasselt: Universiteit Hasselt

Ilse Jonkers

KU Leuven: Katholieke Universiteit Leuven

Benedicte Vanwanseele

KU Leuven: Katholieke Universiteit Leuven

\section{Research}

Keywords: biomechanics, trunk worn IMU , movement quality , hip osteoarthritis , knee osteoarthritis , total knee arthroplasty, level walking

Posted Date: September 17th, 2021

DOI: https://doi.org/10.21203/rs.3.rs-832370/v1

License: (ㅇ) (1) This work is licensed under a Creative Commons Attribution 4.0 International License. Read Full License

Version of Record: A version of this preprint was published at Sensors on April 12th, 2022. See the published version at https://doi.org/10.3390/s22082955. 


\section{Abstract}

Background: This study aimed to evaluate which of movement quality parameters measured with a single trunk worn Inertial Measurement Unit (IMU) can distinguish the gait pattern of people with hip or knee osteoarthritis (OA) compared to asymptomatic controls. Secondly, we evaluated the sensitivity of these parameters to capture gait changes at 6 weeks, 3, 6, and 12 months following total knee arthroplasty (TKA). Thirdly, we investigated whether observed changes in movement quality from 6 weeks and 12 months following total knee arthroplasty were related to changes in patient-reported outcome measures.

Methods: Twenty people with hip OA, 18 people with knee OA, and 20 asymptomatic controls participated in this study. Seventeen people with knee OA were treated with a TKA and additionally followed for one year postoperatively. The participants were equipped with a single trunk-worn IMU and were instructed to walk back and forth along a $10 \mathrm{~m}$ walkway at a self-selected speed. The movement quality parameters (quantified by symmetry-step/stride regularity; complexity-Sample entropy; smoothness-Log Dimensionless Jerk; and dynamic stability-maximum Lyapunov Exponent) were calculated from the raw 3D acceleration signal. Comparisons were made between groups and between timepoints in the TKA patients. Finally, changes in movement quality were correlated with patient-reported outcomes in the TKA group.

Results: We found significant group differences in movement symmetry and stability pre-operatively. Post-TKA, all parameters, except movement smoothness, reflected an initial decrease in movement quality at 6 weeks postTKA, but all except movement complexity, normalised after 6 months. Moreover, improved movement quality (6 weeks-12 months post-TKA) related to improvements in patient-reported outcome measures.

Conclusions: A single lower back IMU can characterise movement quality before and after a total joint arthroplasty. Most symmetry measures recovered but a more unstable, less complex gait pattern was observed at follow-up. The correlation between these parameters and patient-reported outcome measures shows the potential to monitor movement quality in a clinical setting to inform objective data driven personalised rehabilitation of quantified gait symmetry, stability, complexity, and smoothness.

\section{Background}

Hip and knee osteoarthritis (OA) are frequent, disabling musculoskeletal disorders and are a leading cause of lower extremity disabilities[1]. Symptomatic OA is characterised by pain, stiffness, physical disabilities, and difficulties in performing daily life activities[1]. Patient-reported outcome measures (PROM) are most frequently used to measure the impact on everyday functioning, but lack biomechanical insights[2]. However, PROMS are prone to subjective factors whereas we need objective measures to inform rehabilitation that ideally also relate to biomechanical function as this is what we aim to remediate. Thus, knowing about the underlying mechanism of a poorer outcome can help to identify more personalised treatment targets.

Laboratory studies have identified biomechanical changes in the gait pattern of patients with hip or knee OA[3-6]. Normalisation of lower limb biomechanics is often strived for, particularly in the case of joint replacement surgery, and that this measurement can be done with either highly simple (step length) or highly complex (up to the level of loading) parameters. However, an altered gait pattern can still be present up to one year following total knee arthroplasty (TKA) or total hip arthroplasty (THA)[7-11]. Therefore, measuring biomechanical alterations in a clinical setting would be beneficial to monitor the gait pattern. However, to date, conventional methods to monitor 
and evaluate the gait pattern rely on extensive lab-based motion capture systems that do not necessarily reflect movement patterns outside of a lab.

Alternatively, Inertial Measurement Units (IMUs) bear the advantage of easy mounting to the patients without the restriction or complexity of a gait lab[12] and a scoping review by Kobsar et al. (2020) demonstrated the increased popularity of using wearable technology for (mostly spatio-temporal) gait analysis in people with OA[13]. Alternatively, an IMU at the lower trunk level was used as an easy and unobtrusive method to assess gait and running biomechanics, both in healthy adults and patients with neuromuscular disorders[12]. The use of only one sensor allows (as a proxy to) objectively detecting changes in the 3dimensional movement characteristics of the centre of mass (CoM)[14, 15] providing global kinematical information during walking. As such, three different components of gait can be assessed i.e., vertical (VT) acceleration reflecting gait efficiency, mediolateral (ML) acceleration reflecting left-right sway and thus stability and anterior-posterior (AP) acceleration reflecting propulsion and braking.

Dedicated IMU-derived measures, both in the time or frequency domain, have been used to assess movement quality in terms of symmetry, complexity, smoothness, and stability. Previously, researchers used these parameters to determine the variability and knee function in anterior cruciate ligament deficient knees[16-18], gait smoothness in older adults with central dysfunction[19], fall risk in older adults[20, 21], and motor recovery after stroke[22], and Parkinson's disease[23]. Furthermore, these parameters were sensitive enough to discriminate between the gait pattern of healthy controls and pre-manifested individuals with Huntington's (i.e. gene carriers, but not displaying any functional decline)[24] and early gait alteration in patients with Multiple-sclerosis[25]. Moreover, a healthy physiological and biomechanical function is characterised by high complexity that enables adaptability to unpredicted events in daily life[26], an optimal level of stability that attenuates small perturbations and transitions to different motor patterns, as well as a smooth motor pattern that indicates well-trained motor behaviour[22]. Thus, these parameters could be instrumental in assessing the functionality of a patient in a clinical setting and ideally could objectively inform targeted rehabilitation strategies. However, the discriminant ability of the parameters as mentioned above in people with OA from asymptomatic controls is still mostly unexplored, thereby exploring if measured differences represent actual differences that surpass measurement error and thus, related to the OA status. Furthermore, it would be of interest to assess whether these parameters can monitor the impact of a total joint arthroplasty on gait characteristics and the follow-up of the recovery process. The latter is needed to obtain better insights in how the movement quality typically evolves post-TKA. Moreover, the sensitivity of a parameter to change is important for monitoring and diagnostic work. Thus, providing a step towards data informed personalised rehabilitation by showing potential rehabilitation targets that are easy to monitor in a clinical setting.

Therefore, we have a threefold aim: 1) to evaluate which parameters derived from a single trunk worn IMU can distinguish the gait pattern of people suffering from symptomatic hip or knee OA compared to asymptomatic controls; 2) Evaluate the sensitivity of these parameters to capture changes in gait properties at six weeks, three, six, and 12 months following total knee arthroplasty (TKA). 3) Investigated whether observed changes in movement quality parameters between 6 weeks and 12 months following TKA are related to patient-reported functioning, pain, symptoms, sports/recreation, and quality of life.

\section{Materials And Method}




\section{Study design and study sample}

This prospective cohort study was approved by the local ethics committees of the University Hospital Leuven in collaboration with Ziekenhuis Oost-Limburg (Genk) and Jessa Hospital (Hasselt, Belgium) (S59857). The crosssectional analysis consisted of 20 people with unilateral end-stage hip OA, 18 people with unilateral end-stage knee $\mathrm{OA}$ and 20 asymptomatic controls. The inclusion and exclusion criteria are described in Table 1. The eighteen people with knee OA were treated with a TKA, and 17 were re-evaluated at six weeks, three months, six, and 12 months postoperatively (Figure 1). One participant dropped out due to a herniated disc with functional impairment. This study is a secondary analysis of a larger project (S59857) that evaluated the hip and knee joint contact forces in people with degenerative joint disorders and following a total joint arthroplasty. For that study, the sample size was based on the compartmental forces measured in subjects with an instrumental knee prosthesis (1.61+/-0.305 body weight during gait[27]). Assuming that a change in contact forces of one standard deviation to be significant and to achieve a power of 0.8 , a sample of 14 subjects was required. Taking a possible $15-20 \%$ loss of participants into account during the follow-up, we recruited 18 to 20 participants per cohort.

\section{Data acquisition}

We placed a single tri-axial IMU (MVN BIOMECH Awinda, Xsens Technologies, sampling at $60 \mathrm{~Hz}[28]$ ) with a 3D accelerometer at the level of L5/S1 using double-sided tape. We used an additional Velcro strap around the participant's waist to further secure the IMU and minimise excessive movement. Subjects were instructed to walk at self-selected speed in a straight line of $10 \mathrm{~m}$ across our movement laboratory (MALL, KUL, Belgium) at different

Table 1

Inclusion and exclusion criteria for the participants

evaluation points. People with hip OA were only measured once (pre-THA), and the people with knee OA were evaluated five times (pre-TKA, six weeks, three months, six, and 12 months post-TKA) (see Figure 1). The asymptomatic controls returned to the MALL for a re-evaluation. We used the data from asymptomatic controls to calculate the minimal detectable change using the interclass correlation coefficient (ICC) of the movement quality parameters. The Hip disability (Hip OA subjects) and Knee injury Osteoarthritis Outcome Score (Knee OA and Asymptomatic subjects) (HOOS and KOOS) were completed to evaluate patient reported outcome measures. Figure 1 gives the flow of the data collection and the number of participants measured at each time instance.

\section{Data processing}

For each walking trail, the sensor tilt was corrected to convert the accelerations from the local sensor XYZcoordinate system to the global anterior-posterior (AP), vertical (VT), and mediolateral (ML) coordinate system[29]. After that, the AP and ML accelerations were used to identify the individual left and right steps[14]. The steady-state steps[30] were extracted and concatenated to create one long, continuous time series[31]. Considering that the stability and complexity measures are sensitive to the time series length used as input, we used a fixed-step approach to establish the length of the time-series[32]. We determined the least 
Healthy population

Inclusion

- Aged between 50 - 75 years old

- Understand the Dutch language

- Able to walk $10 \mathrm{~m}$ and ascent/descent the stairs

\section{Exclusion}

- Diagnosed with musculoskeletal or neurological disorders

- Pain in hips, knees or ankles, which affect normal movement
Patient population

Inclusion

- Aged between 50 - 75 years old

- Diagnosed with hip or knee OA

- Awaiting of total hip of knee replacement surgery

- Understand the Dutch language

- Able to walk $10 \mathrm{~m}$ and ascent/descent the stairs

Exclusion

- Corticosteroid injection 3 months before inclusion to the study

- Joint replacement in other lower limb joints

- Diagnosed with symptomatic hip or knee OA on the contralateral knee

- Symptomatic degenerative disorders in other lower limb joints

- Neurological conditions that could alter movement pattern

- History of pathological osteoporotic fractures (in hip, knee or ankle joints)

number of steps taken by the participants and truncated the signal length of all other participants to that number of steps ( $n=47$ which corresponds to $+/-1500$ samples).

Movement quality was then evaluated in terms of: (1) movement symmetry, (2) local dynamic stability (3) movement complexity, and (4) movement smoothness. First, movement symmetry was quantified as step and stride regularity. These were calculated using the first two dominant peaks after the zero phase of the unbiased autocorrelation with perfect symmetry equal to one[15]. Since a cyclic signal will produce an autocorrelation with peak values with a time lag equivalent to the period of the signal, the first and second dominant peak represents phase shifts equal to one step and one stride, respectively[15]. The unbiased autocorrelation

signal was normalised to equal one at zero phase shift. Therefore, the height of the first dominant peak shows the autocorrelation coefficient between consecutive steps, and the height of the second dominant peak the autocorrelation coefficient between consecutive strides and is therefore considered a symmetry index. Since ML trunk accelerations produce both positive and negative values representing left-right trunk sway, step regularity in $\mathrm{ML}$ direction are always negative. Therefore, the absolute values are used for analysis. In both cases, lower values of the autocorrelation coefficient indicate more asymmetry.

In the second category, local dynamic stability was calculated by estimating the short-term and long-term maximum Lyapunov Exponent (LyE $\lambda_{S}$ and LyE $\lambda_{\mathrm{L}}$, respectively). The LyE captures how a system responds to perturbations by calculating the divergence of nearest neighbours in state spaces using Rosenstein's method[33] and as proposed by Bruijn et al. (2010)[34]. For the calculation of the LyE, we set the embedding dimension to 
5[34]. The time delay was calculated per subject as the decrease in the autocorrelation curve of 1-1/e as this retained the smallest errors[33]. LyE is calculated over two time-increments: LyE $\lambda_{S}$ over 0-0.5 strides and LyE $\lambda_{L}$ over 4-10 strides. The $\lambda_{S}$ indicates how well the systems deal with perturbations at the step or stride level, whereas $\lambda_{L}$ evaluates how the system is able to handle perturbations over the time increment of several strides. Higher values indicate lower dynamic stability[34,35], indicating an unstable gait pattern with a higher risk of falling[35].

The third and fourth categories are the movement complexity and movement smoothness measure; these are quantified as sample entropy and log dimensionless jerk (LDLJ-A), respectively. Sample entropy captures waveform predictability with higher values indicating less periodicity, thus, more unpredictability[36]. We used nonlinear mathematical algorithms previously described by Richman and Moorman (2000)[37]. As input for the calculation of the sample entropy we used the time series sample length $(\mathrm{N})$ corresponding to the least number of steps taken as described previously, the series length $(\mathrm{m})$ of 2 data points, and a tolerance window ( $r$ ) normalised to 0.2 times the standard deviation of the timeseries[36].

LDLJ-A assesses movement smoothness by quantifying the changes in the acceleration signal (jerk-a derivative of the acceleration signal) as proposed by Melendez-Calderon et al. (2021)[38]. The Euclidean norm (2-norm) of the acceleration signals (i.e., Pythagorean Theorem over acceleration in VT, ML, and AP direction) was used to calculate the LDLJ-A over each step separately, thereafter the average was calculated to obtain a single smoothness measure per subject. A signal that shows minimal changes in the acceleration and deceleration pattern is considered smoother. A smoother movement is indicated by lower values[22].

All data were processed and analysed using customised MATLAB scripts (MATLAB 2018b, The Math Works, Inc. Natick, Massachusetts, USA).

\section{Statistical analysis}

Data were not normally distributed as assessed by visual inspection of the histogram, Q-Q plot, and Shapiro-Wilk test. Therefore, non-parametric statistics were used. We used the Mann-Whitney U test for group differences between asymptomatic controls and people with hip OA and between asymptomatic controls and people with knee OA. The Minimal Detectable Change (MDC) per dependent variable was calculated using data from the testretest of all asymptomatic controls using the interclass correlation coefficient $(\operatorname{ICC}(3, \mathrm{k}))[39]$. The MDC was calculated to check whether a difference between the two cohorts is a fundamental difference that surpasses the system's measurement errors.

To assess how the parameters evolve after a TKA, Friedman's chi-square ANOVA was conducted. When a significant main effect was found $(a<0.05)$, a Wilcoxon signed-rank test with a Bonferroni correction $(a<0.005)$ was calculated to test for differences between timepoints. A spearman's rho correlation coefficient was calculated on the change scores between 6 weeks and 12 months post-op to relate changes in movement quality to patientreported functioning, symptoms, and quality of life. A spearman's rho correlation coefficient between 0-0.25 was considered low, from 0.25-0.5 fair, 0.5-0.75 moderate, and 0.75-1.0 high. Statistical analysis was performed using Python SciPy statistics package (v1.4.1) and missing data were omitted[40].

This resulted in preoperative cohorts of 20 asymptomatic controls, 18 people with knee OA and 20 people with hip OA. Seventeen people post-TKA were included in the follow-up analysis (one drop-out at 12 months) and in the 
correlation analysis between movement quality and patient reported pain, symptoms, ADL, and QOL. The correlation analyses of patient reported sports/recreation was on 15 people (drop-out of 3 due to an inability to answer the questionnaire).

\section{Results}

\section{Preoperative cohort comparison}

Subject characteristics are shown in table 2. The average step-time is longer for both OA cohorts than the asymptomatic cohort. Patient-reported outcomes (PROMS) were significantly worse in the OA cohorts than in the healthy controls (Table 2). The PROMS following TKA improved over time but remained significantly lower than the asymptomatic controls (Table 2 ).

Table 2

Participant characteristics mean (SD)

\begin{tabular}{|c|c|c|c|c|c|c|c|}
\hline & Healthy & Hip OA & Knee OA & Knee OA & Knee OA & Knee OA & Knee OA \\
\hline & to & to & to & $\mathrm{t} 1$ & t2 & t3 & $\mathrm{t} 4$ \\
\hline mass (kg) & $\begin{array}{l}70.8 \\
(14.2)\end{array}$ & $\begin{array}{l}75.4 \\
(11.6)\end{array}$ & $\begin{array}{l}79.8 \\
(8.2)\end{array}$ & 79.8 (8.5) & & & \\
\hline Height (m) & $\begin{array}{l}1.70 \\
(0.08)\end{array}$ & $\begin{array}{l}1.75 \\
(0.09)\end{array}$ & $\begin{array}{l}1.75 \\
(0.08)\end{array}$ & $1.75(0.08)$ & & & \\
\hline age (years) & $\begin{array}{l}62.7 \\
(8.5)\end{array}$ & $\begin{array}{l}63.1 \\
(6.2)\end{array}$ & $\begin{array}{l}65.1 \\
(5.1)\end{array}$ & 64.7 (4.9) & & & \\
\hline Gender (M/F) & $9 / 11$ & $11 / 9$ & $11 / 7$ & $10 / 7$ & & & \\
\hline Step Time (s) & $\begin{array}{l}31.16 \\
(2.01)\end{array}$ & $\begin{array}{l}33.10 \\
(2.31)\end{array}$ & $\begin{array}{l}33.65 \\
(2.5)\end{array}$ & 35.53 (3.64) & $\begin{array}{l}33.82 \\
(2.27)\end{array}$ & $\begin{array}{l}32.71 \\
(2.11)\end{array}$ & $\begin{array}{l}32.71 \\
(1.99)\end{array}$ \\
\hline \multicolumn{8}{|l|}{$\begin{array}{l}\text { Patient-reported } \\
\text { outcomes }\end{array}$} \\
\hline Pain & $\begin{array}{l}95.35 \\
(6.1)\end{array}$ & $\begin{array}{l}50.85 \\
(11.85)\end{array}$ & $\begin{array}{l}49.77 \\
(13.89)\end{array}$ & $54.34(14.89)$ & $\begin{array}{l}63.25 \\
(16.04)\end{array}$ & $\begin{array}{l}78.99 \\
(16.52)\end{array}$ & $\begin{array}{l}78.04 \\
(14.43)\end{array}$ \\
\hline Symptoms & $\begin{array}{l}96.9 \\
(5.5)\end{array}$ & $\begin{array}{l}52.25 \\
(17.9)\end{array}$ & $\begin{array}{l}50.89 \\
(22.90)\end{array}$ & $52.32(12.02)$ & $\begin{array}{l}59.82 \\
(13.97)\end{array}$ & $\begin{array}{l}69.34 \\
(16.00)\end{array}$ & $\begin{array}{l}80.06 \\
(13.83)\end{array}$ \\
\hline $\begin{array}{l}\text { Activities of daily } \\
\text { life }\end{array}$ & $\begin{array}{l}98.7 \\
(2.55)\end{array}$ & $\begin{array}{l}56.4 \\
(15.45)\end{array}$ & $\begin{array}{l}60.17 \\
(17.92)\end{array}$ & $62.38(14.03)$ & $\begin{array}{l}73.15 \\
(16.13)\end{array}$ & $\begin{array}{l}82.52 \\
(14.94)\end{array}$ & $\begin{array}{l}87.38 \\
(12.66)\end{array}$ \\
\hline Sport & $\begin{array}{l}93.3 \\
(9.2)\end{array}$ & $\begin{array}{l}24.05 \\
(23.2)\end{array}$ & $\begin{array}{l}29.44 \\
(26.64)\end{array}$ & $18.96(21.25)$ & $\begin{array}{l}31.18 \\
(16.65)\end{array}$ & $\begin{array}{l}47.81 \\
(25.32)\end{array}$ & $\begin{array}{l}55.31 \\
(27.00)\end{array}$ \\
\hline Quality of life & $\begin{array}{l}92.05 \\
(9.8)\end{array}$ & $\begin{array}{l}27.95 \\
(15.85)\end{array}$ & $\begin{array}{l}31.25 \\
(17.06)\end{array}$ & 39.58 (17.34) & $\begin{array}{l}42.71 \\
(15.27)\end{array}$ & $\begin{array}{l}57.29 \\
(16.61)\end{array}$ & $\begin{array}{l}61.98 \\
(17.16)\end{array}$ \\
\hline
\end{tabular}

Movement symmetry measured by the step regularity was different between cohorts. People with hip OA had a more asymmetrical gait pattern than asymptomatic controls in all three directions (Figure 2 and Table 3). All 
these differences exceeded the minimal detectable change threshold (Figure 2). People with knee OA also displayed an asymmetrical gait pattern, however only in the VT and ML direction. These differences in people with knee OA exceeded the minimal detectable change (Figure 2).

Long-term local dynamic stability over the strides (LyE $\left.\lambda_{L}\right)$ was not different in people with hip or knee OA compared to controls (Figure 3 and Table 3). Short-term local dynamic stability (LyE $\lambda_{S}$ ) was significantly lower in people with knee OA in all three directions, with in AP direction surpassing the minimal detectable change. Additionally, a significantly lower LyE $\lambda_{S}$ in $M L$ direction and bordering on significance level in AP direction was found between people with hip OA and asymptomatic controls, but the difference was too small to surpass the minimal detectable change. No group differences were found for either Sample Entropy (Figure 3) or LDLJ-A (Figure 4).

Table 3

Test statistics group comparison. 
Asymptomatic controls

$\begin{array}{llll}\text { avg } & \text { avg } & \text { ICC } & \text { MDC }\end{array}$
Hip OA

avg Diff
Knee OA

avg Diff
$P$
value

VT

\begin{tabular}{lllllllllll} 
StepRegularity & 0.78 & 0.77 & 0.89 & 0.05 & 0.64 & $\mathbf{0 . 1 3}$ & $<0.001$ & 0.70 & $\mathbf{0 . 0 8}$ & $<0.01$ \\
\hline StrideRegularity & 0.71 & 0.71 & 0.79 & 0.12 & 0.63 & 0.08 & $\mathbf{0 . 0 2}$ & 0.69 & 0.02 & 0.23 \\
\hline SampEn & 0.77 & 0.85 & 0.75 & 0.20 & 0.73 & 0.05 & 0.08 & 0.75 & 0.03 & 0.21 \\
LyE $\ddot{e}_{S}$ & 3.57 & 3.47 & 0.70 & 0.85 & 3.31 & 0.27 & 0.09 & 3.05 & 0.52 & $<0.01$ \\
LyE $\ddot{e}_{L}$ & 0.03 & 0.03 & 0.02 & 0.06 & 0.02 & 0.00 & 0.26 & 0.03 & 0.00 & 0.45
\end{tabular}

ML

$\begin{array}{lllllllllll}\text { StepRegularity } & -0.55 & -0.55 & 0.79 & 0.15 & -0.38 & \mathbf{0 . 1 7} & <0.01 & -0.41 & 0.14 & <0.001 \\ \text { StrideRegularity } & 0.46 & 0.48 & 0.49 & 0.27 & 0.42 & 0.04 & 0.16 & 0.44 & 0.02 & 0.31 \\ \text { SampEn } & 0.91 & 0.95 & 0.80 & 0.13 & 0.87 & 0.04 & 0.31 & 0.97 & 0.07 & 0.07 \\ \text { LyE ë } & 3.65 & 3.67 & 0.80 & 0.56 & 3.32 & 0.33 & \mathbf{0 . 0 4} & 3.23 & 0.42 & \mathbf{0 . 0 2} \\ \text { LyE } \ddot{e}_{\mathrm{L}} & 0.02 & 0.02 & -0.41 & 0.07 & 0.02 & 0.00 & 0.46 & 0.02 & 0.00 & 0.37\end{array}$

AP

StepRegularity

0.70

$\begin{array}{lll}0.70 & 0.87 & 0.05\end{array}$

0.60

$\begin{array}{ll}0.10 & 0.02\end{array}$

$\begin{array}{lll}0.68 & 0.02 & 0.28\end{array}$

StrideRegularity

0.63

0.61

0.78

0.11

0.63

$0.00 \quad 0.48$

$\begin{array}{lll}0.67 & 0.04 & 0.12\end{array}$

SampEn

0.58

0.60

$0.91 \quad 0.09$

0.64

$0.06 \quad 0.06$

$\begin{array}{lll}0.60 & 0.02 & 0.20\end{array}$

LyE $\ddot{e}_{S}$

3.29

3.28

$0.86 \quad 0.38$

3.01

$0.27 \quad 0.05$

2.78

$0.51<0.01$

LyE $\ddot{e}_{\mathrm{L}}$

0.03

0.03

$-0.86$

0.10

0.03

0.00

0.20

0.03

0.00

0.29

\section{Norm vector}

LDLJ

$-6.17$

$\begin{array}{ll}-6.10 & 0.90\end{array}$

0.14

$\begin{array}{lll}-6.23 & 0.06 & 0.24\end{array}$

$\begin{array}{lll}-6.16 & 0.00 & 0.48\end{array}$

Averages of test (avg t0) and retest (avg t1) of the asymptomatic controls with the associated interclass correlation coefficient (ICC) and the minimal detectable change (MDC). The averages for the hip OA and knee OA subjects are reported, with the difference in mean (Diff) between the healthy cohort and an OA cohort. All significant $p$-values (<0.05) and differences that surpass the MDC are in bold

\section{Longitudinal follow-up following TKA}

Some changes in asymmetry can be observed at the re-evaluation moments (Figure 5). A trend towards increased step asymmetry was observed in VT and ML direction from pre-op to six weeks post-OP, followed by a significant 
improvement in step regularity in VT direction at six- and 12-months post-OP compared to six weeks post-op. Step regularity in the ML direction after TKA remained more asymmetrical than the asymptomatic controls one-year post-OP.

Short and long-term dynamic stability measures did not significantly change over time following TKA. However, we found significantly higher LyE $\lambda_{\mathrm{L}}$ values than the asymptomatic controls at 12-month post-op (Figure 6) indicating lower dynamic stability at that timepoint. Furthermore, the LyE $\lambda_{s}$ in $M L$ and AP direction was significantly lower compared to asymptomatic controls, reflecting a more rigid movement pattern. The LyE $\lambda s$ in the VT direction was no longer significantly different from asymptomatic controls at 12 months post-op.

There was a reduction in movement complexity (sample entropy) in the VT and ML direction at six weeks post-OP compared to pre-OP complexity values (Figure 6). Sample entropy in the ML direction significantly improved at six months post-OP; however, this improvement was not observed in the VT direction. Movement smoothness quantified as LDLJ-A was not affected by the TKA procedure (Figure 7).

\section{Correlations}

Only step regularity in AP direction was fairly correlated $(r=0.48)$ with quality of life, showing that an increase in step symmetry correlated with an increase in patient-reported quality of life (Table 4).

Improvements in stability measures ( $L y E \lambda_{S}$ and $\lambda_{L}$ ) were fairly to moderately correlated with improvements in patient-reported pain, quality of life, $A D L$, and sports and recreation. An increase in $L y E \lambda_{S}$ in $M L$ and $A P$ direction (i.e. a trend towards values found in healthy controls) reflected decreases in pain ( $r=0.51$ and $r=0.54)$, increase in the ability to perform daily life activities ( $r=0.43$ and 0.55$)$ and quality of life ( $r=0.43$ and $r=0.48)$. Indicating that a positive change in short term stability (more resembling asymptomatic controls) related to better patient-reported outcome scores. Changes in LyE $\lambda_{\mathrm{L}}$ in AP and $\mathrm{ML}$ direction were negatively correlated with changes in sports/recreation ( $r=-0.56$ and $r=-0.4)$, reflecting a decrease in $L y E \lambda_{L}$ towards values measured in healthy controls related to better patient-reported outcomes in sport and recreation. Moreover, we found that a decrease in LyE $\lambda_{L}$ was also fairly correlated with a decrease in symptoms.

Lastly, increases in sample entropy values in ML direction was positively correlated with an increase in patientreported quality of life. Similarly, to our short-term stability measures, an increase in sample entropy reflected a trend towards values found in our asymptomatic cohort. All correlation coefficients and significant values can be found in Table 4.

Table 4

Correlations between movement quality parameters and patient reported functioning 


\begin{tabular}{|c|c|c|c|c|c|c|c|c|c|c|}
\hline & \multicolumn{2}{|l|}{ Pain } & \multicolumn{2}{|l|}{$A D L$} & \multicolumn{2}{|l|}{ QOL } & \multicolumn{2}{|c|}{ Symptoms } & \multicolumn{2}{|c|}{ Sports/rec } \\
\hline & rho & ${ }_{\text {value }}^{p-}$ & rho & ${ }_{\text {value }}^{p-}$ & rho & $\begin{array}{l}p- \\
\text { value }\end{array}$ & rho & $\begin{array}{l}p- \\
\text { value }\end{array}$ & rho & $\begin{array}{l}p- \\
\text { value }\end{array}$ \\
\hline \multicolumn{11}{|l|}{ VT } \\
\hline StepRegularity & -0.15 & 0.55 & -0.28 & 0.28 & 0.06 & 0.83 & -0.32 & 0.21 & -0.40 & 0.13 \\
\hline StrideRegularity & -0.11 & 0.69 & 0.05 & 0.84 & 0.16 & 0.55 & -0.01 & 0.96 & -0.16 & 0.57 \\
\hline SampEn & 0.19 & 0.46 & 0.18 & 0.49 & 0.09 & 0.74 & 0.28 & 0.27 & -0.16 & 0.57 \\
\hline LyE ës & 0.34 & 0.19 & 0.34 & 0.18 & 0.38 & 0.14 & 0.16 & 0.55 & 0.05 & 0.86 \\
\hline LyE ë & -0.08 & 0.76 & -0.04 & 0.87 & -0.14 & 0.59 & -0.43 & 0.09 & -0.08 & 0.77 \\
\hline \multicolumn{11}{|l|}{ ML } \\
\hline StepRegularity & -0.00 & 0.99 & 0.07 & 0.78 & 0.09 & 0.74 & -0.01 & 0.98 & -0.27 & 0.32 \\
\hline StrideRegularity & 0.04 & 0.89 & 0.18 & 0.50 & 0.08 & 0.77 & 0.27 & 0.29 & -0.25 & 0.37 \\
\hline SampEn & 0.11 & 0.69 & 0.05 & 0.85 & 0.47 & 0.05 & 0.24 & 0.36 & 0.09 & 0.76 \\
\hline LyE ës & 0.51 & 0.03 & 0.43 & 0.08 & 0.43 & 0.09 & 0.15 & 0.56 & -0.05 & 0.85 \\
\hline LyE $\ddot{e}_{\mathrm{L}}$ & -0.10 & 0.71 & -0.02 & 0.93 & -0.39 & 0.12 & -0.17 & 0.52 & -0.56 & 0.03 \\
\hline \multicolumn{11}{|l|}{ AP } \\
\hline StepRegularity & 0.28 & 0.28 & 0.26 & 0.32 & 0.48 & 0.05 & -0.08 & 0.76 & -0.05 & 0.85 \\
\hline StrideRegularity & -0.06 & 0.82 & 0.19 & 0.46 & 0.15 & 0.58 & 0.11 & 0.66 & -0.26 & 0.34 \\
\hline SampEn & 0.00 & 0.99 & -0.27 & 0.30 & -0.22 & 0.40 & -0.23 & 0.37 & -0.09 & 0.75 \\
\hline LyE ës & 0.54 & 0.03 & 0.55 & 0.02 & 0.48 & 0.05 & 0.28 & 0.28 & 0.11 & 0.69 \\
\hline LyE ë & -0.17 & 0.52 & 0.13 & 0.62 & -0.07 & 0.78 & -0.10 & 0.69 & -0.40 & 0.14 \\
\hline \multicolumn{11}{|l|}{ 2-norm } \\
\hline LDLJ & -0.05 & 0.85 & 0.06 & 0.82 & 0.10 & 0.71 & 0.15 & 0.57 & -0.51 & 0.05 \\
\hline
\end{tabular}

Moderate and significant correlations are in bold

\section{Discussion}

This study had a threefold aim: 1) to evaluate which movement quality parameters derived from a single trunk worn IMU can distinguish the gait pattern of people suffering from symptomatic hip or knee OA compared to asymptomatic controls; 2) Evaluate the sensitivity of these parameters to capture changes in gait properties at six weeks, three, six, and 12 months following total knee arthroplasty (TKA). 3) Investigated whether observed changes in movement quality parameters between 6 weeks and 12 months following TKA are related to patientreported functioning, pain, symptoms, sports/recreation, and quality of life. Based on this study's findings, a single trunk worn IMU can be used in the assessment of gait quality in people with OA. The parameters of interest 
between the people with $\mathrm{OA}$ and the asymptomatic controls are symmetry and stability. The results furthermore show that symmetry, stability, and complexity are of interest to monitor in the follow-up of people treated with a TKA. The results are a step in the direction towards data informed rehabilitation strategies.

To reach our first aim, we found that of all studied parameters, we found a more asymmetric gait pattern in people with hip OA as well as a more asymmetrical and rigid gait pattern in people with knee OA compared to asymptomatic controls. However, only the symmetry but not the stability measures surpassed the minimal detectable change, which indicates that the differences between the groups surpass naturally occurring betweensession variation and measurement error. Therefore, we can confidently state that symmetry measures can detect differences in movement quality between people with OA and asymptomatic controls.

Gait symmetry was significantly lower in both hip and knee OA patients compared to asymptomatic individuals. These results corroborate previous research finding gait asymmetry in people with unilateral OA[41-43]. This compensatory gait pattern (i.e., more asymmetry) might also lead to a higher risk of developing OA in other joints as aberrant mechanical loading is a risk factor to the onset and progression of $O A[5]$.

Short term dynamic stability (LyE) was not significantly affected in people with hip OA; whereas, in people with knee OA short term dynamic stability values in all three directions were significantly lower compared to asymptomatic controls. This finding was surprising since higher maximum Lyapunov exponents during walking have been associated with an increased fall risk[35]. However, when we consider stability in the construct of having an optimum (e.g., healthy people), then both extremes either significantly higher and significantly lower are undesirable. The maximum Lyapunov Exponent is calculated as the maximal deviation of the nearest neighbours in an attractor state, in our case, steady-state walking. On the one hand of the extreme, when the LyE is significantly increased, the system can be considered unstable and is less able to handle perturbations (i.e., small perturbation will knock the system off course), i.e., an undesirable outcome. Alternatively, when the LyE is significantly smaller, might reflect a too rigid system i.e., reflecting a limited adaptability to change motor pattern (e.g., changing from straight line walking to sidestepping an unanticipated obstacle); considering we found lower LyE values in the people with knee OA, we theorise that people with knee OA adapt a more rigid movement pattern to ensure stability. However, we need to investigate this phenomenon and its consequences in more detail in future research.

Unlike gait stability and symmetry, gait complexity and smoothness did not significant differ between either hip nor knee OA and asymptomatic controls. This means that the gait pattern of people with OA are still quantified by a healthy physiological biomechanical pattern and are indicative of well-trained motor behaviour.

To evaluate the sensitivity of the movement quality parameters to capture gait changes at four re-evaluation moments post-TKA, we found that by using a single trunk worn IMU changes in symmetry, stability and complexity can be detected. Following TKA, we observe an initial deterioration in symmetry, followed by a significant improvement after six months. These significant improvements in symmetry resulted in a normalisation of these values-at 12 months post-TKA, the group was no longer significantly different from asymptomatic controls.

We observed a similar pattern in short-term dynamic stability, an initial (non-significant) further decrease followed by an increase at 6 and 12 months. However, only in vertical direction, at 12-months post-TKA, there is no longer a significant difference with asymptomatic controls. Additionally, we found a gradual rise of the long-term stability;

Page $12 / 26$ 
this rise was too small to be significantly different between re-evaluation moments. However, it resulted in a significantly larger long-term LyE, thus less able to handle small perturbations over multiple strides, at 12 months post-TKA compared to asymptomatic controls. Even though only the short-term LyE was associated with the risk of falling[35], Su \& Dingwell (2007) argued that although the long- term maximum Lyapunov Exponent did not predict fall risk, it does quantify inherent stability[44]. Therefore, from our results, we theorise that over a time increment of a single step, people post-TKA adopt a more rigid movement pattern to ensure stability; however, that might compromise the dynamic stability over the time increment of several strides by limiting the adaptability of the system.

Moreover, we observe an initial decrease in movement complexity (sample entropy) at six weeks post-OP that shows a trend toward normalisation after six months. A healthy gait pattern is characterised by a high degree of complexity to adapt to unpredictable events $[17,45]$. Therefore, the drop in movement complexity leads to a gait pattern that is less adaptable to unexpected changes and is less able to use step-to-step adjustments to regulate balance control effectively[17,46], which, in turn, could lead to an increased risk of falling. Contrastingly, movement smoothness did not seem affected by the TKA as we observed changes nor trends between the timepoints. Concluding that movement smoothness quantified by the log dimensionless jerk is not a sensitive to biomechanical gait changes.

The study's third aim was to investigate whether observed changes in movement quality parameters between 6 weeks and 12 months following TKA are related to patient-reported functioning, pain, symptoms, sports/recreation, and quality of life. Based on the results we found some fair to moderate correlations between changes in movement quality and changes in patient reported functioning.

The most notable are the significant corelations between stability and patient reported pain, quality of life, performing activities of daily living, and sports/recreation. In fact, a larger objectively measured increase in shortterm LyE in ML and AP direction from 6 weeks to 12 months post-TKA (thus more towards values reported in asymptomatic controls) was correlated with a larger recovery in patient-reported functioning in daily life activities, less hinder from pain and a better quality of life. The quality-of-life section of the KOOS mainly reflects how well people can "trust" their affected knee (i.e., giving away). Thus, changes in objectively measured stability appear to correlate with changes in subjective feelings of being stable. Similar to the correlations found in the short-term $\mathrm{LyE}$, a more considerable increase in sample entropy in $\mathrm{ML}$ direction was related to a more considerable improvement in patient-reported quality of life. Reflecting that a higher complexity relates to a better quality of life. This could reflect the hypothesis that ML motion is under a direct feedback control loop for step-by-step adjustments for effective balance control[46]. Thus, higher complexity (i.e., towards more healthy values) might be related to a better balance control and thus relate to a better quality of life.

All our findings combined (i.e., initial decrease in quality followed by a recovery and normalisation) show the potential of a single IMU to follow-up the recovery following TKA. The correlations found between improvements in symmetry, stability and complexity measures and improvements in patient-reported outcomes could identify objective treatment targets (e.g., increase stability) to improve patient-reported functioning. Future work should determine if incorporating these objective measures in clinical practice ensuring a better and more personalised rehabilitation plan leads to improved functioning of these people.

\section{Limitations}


There are some limitations to this work. Foremost, the sample entropy and the maximum Lyapunov Exponent are sensitive to the number of data points used in the analysis. We tried to overcome this by including the same number of steps for each individual. However, this does indicate that the absolute number reported here cannot be compared to the values reported in the literature. TenBroek et al. (2007) showed that the LyE value increases with fewer data points and that the value stabilises after 5000 samples[47]. Similarly, Yentes et al. (2012) proved that the sample entropy stabilises after 2000 samples[36]. In this study, the number of data points is around 1500 samples, below the 5000- and 2000-sample threshold for the LyE and sample entropy, respectively. Therefore, we most likely overestimated the absolute values of the LyE and sample entropy. However, because we used the same number of steps for each individual, we believe that we can compare the values between our different groups, just not with previously reported work. Furthermore, we used straight line walking within a controlled labbased setting. Although we found significant differences between our groups and following TKA, results might be more pronounced in more complex exercises like ascending or descending stairs or turning. Future research should incorporate more complex daily life activities to gain more insights in symmetry, stability, complexity, and smoothness during daily life activities in people with OA and following TKA. Moreover, we only included a limited number of subjects in this study. Considering that this is a secondary analysis of a larger study, sample size was based on compartmental forces of the knee, which are not comparable to the parameters used within this work. Post-hoc sample size calculation indeed showed that we were slightly underpowered for sample entropy and the maximum Lyapunov Exponent to distinguish the gait pattern of hip OA people from asymptomatic controls. Recognising the ease with which these parameters can be collected, future research should include more subjects to investigate in combination with incorporating these movement quality parameters in a rehabilitation, ensuing data informed personalised rehabilitation targets, lead to an improved function in people with either hip or knee OA and following a total knee arthroplasty.

\section{Conclusions}

A single lower back IMU can be used to characterise movement quality before and after a total joint arthroplasty without the restrictions of a gait lab. We found a more asymmetric gait pattern in people with hip OA as well as a more asymmetrical and rigid gait pattern in people with knee OA compared to asymptomatic controls. Concluding that symmetry and stability are measures of interest in people with OA. We also found that symmetry, stability, and complexity are sensitive to biomechanical changes post-TKA. Showing the ability to objectively monitor time sensitive changes in movement quality after a total joint arthroplasty. Moreover, the correlations found between improvements in symmetry, stability and complexity measures and improvements in patient-reported outcomes could possibly identify objective treatment targets (e.g., increase stability) to improve patient-reported functioning. Future work should determine if incorporating these objective measures in clinical practice, ensuring data driven personalised rehabilitation, leads to an improved functioning of people with (either hip or knee) OA and after a total knee arthroplasty.

\section{Abbreviations}

- OA Osteoarthritis

- TKA Total knee arthroplasty

- IMU Inertial measurement unit

- PROM Patient-reported outcome measures 
- LDLJ Log dimensionless jerk

- LyE $\lambda_{S}$ Short-term maximum Lyapunov exponent

- LyE $\lambda_{\mathrm{L}}$ Long-term maximum Lyapunov exponent

- VT Vertical

- ML Medio-lateral

- AP Anterior-posterior

- Post-OP Postoperatively

- KOOS Knee Injury Osteoarthritis Outcome Score

- HOOS Hip disability Osteoarthritis Outcome Score

- MDC Minimal detectable change

\section{Declarations}

\section{Ethics approval and consent to participate}

This study was approved by the local ethics committees of the University Hospital Leuven in collaboration with Ziekenhuis Oost-Limburg (Genk) and Jessa Hospital (Hasselt, Belgium) (S59857) and all subjects provided written informed consent before participation.

\section{Consent for publication}

Not applicable

\section{Availability of data and materials}

The data that support the findings of this study are not openly available due to GDPR guidelines (i.e., human motion data), and are available from the corresponding author upon reasonable request.

\section{Competing interests}

The authors declare that they have no competing interests

\section{Funding}

This research was funded by Research Foundation Flanders (FWO) grant number T004716N.

\section{Authors' contributions}

Funding acquisition: BV, LDB, IJ, AT, KC.

Study concept and design: JE, BV, AT 
Data collection and analysis: JE, RVDS

Results interpretation: JE, BV, AT, IJ, SVR

Writing - original draft: JE, LDB, KC.

Writing - review and editing: All authors

\section{Acknowledgements}

Not applicable

\section{References}

1. Woolf AD, Pfleger B. Burden of major musculoskeletal conditions. Bull World Health Organ. 2003;81:646-56.

2. Hodt-Billington C, Helbostad JL, Vervaat W, Rognsvåg T, Moe-Nilssen R. Changes in gait symmetry, gait velocity and self-reported function following total hip replacement. J Rehabil Med. 2011;43:787-93.

3. Mills K, Hunt MA, Ferber R. Biomechanical deviations during level walking associated with knee osteoarthritis: A systematic review and meta-analysis. Arthritis Care Res (Hoboken) [Internet]. John Wiley \& Sons, Ltd; 2013 [cited 2020 Sep 4];65:n/a-n/a. Available from: http://doi.wiley.com/10.1002/acr.22015.

4. Meyer CAG, Corten K, Fieuws S, Deschamps K, Monari D, Wesseling M, et al. Biomechanical Gait Features Associated With Hip Osteoarthritis: Towards a Better Definition of Clinical Hallmarks. 2015;1498-507.

5. Felson DT. Osteoarthritis as a disease of mechanics. Osteoarthr Cartil [Internet]. NIH Public Access; 2013 [cited 2019 Jun 6];21:10-5. Available from: http://www.ncbi.nlm.nih.gov/pubmed/23041436.

6. Saxby DJ, Lloyd DG. Osteoarthritis year in review 2016: mechanics. Osteoarthr Cartil [Internet]. Elsevier Ltd; 2017;25:190-8. Available from: http://dx.doi.org/10.1016/j.joca.2016.09.023.

7. Kolk S, Minten MJM, Van Bon GEA, Rijnen WH, Geurts ACH, Verdonschot N, et al. Gait and gait-related activities of daily living after total hip arthroplasty: A systematic review. Clin Biomech [Internet]. Elsevier Ltd; 2014;29:705-18. Available from: http://dx.doi.org/10.1016/j.clinbiomech.2014.05.008.

8. Ewen AM, Stewart S, St Clair Gibson A, Kashyap SN, Caplan N. Post-operative gait analysis in total hip replacement patients $-\mathrm{A}$ review of current literature and meta-analysis. Gait Posture [Internet]. Elsevier B.V.; 2012;36:1-6. Available from: http://linkinghub.elsevier.com/retrieve/pii/S096663621100840X.

9. Foucher KC, Hurwitz DE, Wimmer MA. Preoperative gait adaptations persist one year after surgery in clinically well-functioning total hip replacement patients. J Biomech Elsevier. 2007;40:3432-7.

10. Milner CE. Is gait normal after total knee arthroplasty? Systematic review of the literature. J. Orthop. Sci. Springer Japan; 2009. p. 114-20.

11. Levinger P, Menz HB, Morrow AD, Perrott MA, Bartlett JR, Feller JA, et al. Knee biomechanics early after knee replacement surgery predict abnormal gait patterns 12 months postoperatively. J Orthop Res [Internet]. John Wiley \& Sons, Ltd; 2012 [cited 2020 Jun 8];30:371-6. Available from: http://doi.wiley.com/10.1002/jor.21545.

12. Benson LC, Clermont CA, Bošnjak E, Ferber R. The use of wearable devices for walking and running gait analysis outside of the lab: A systematic review. Gait Posture. Elsevier B.V.; 2018. pp. 124-38. 
13. Kobsar D, Masood Z, Khan H, Khalil N, Kiwan MY, Ridd S, et al. Wearable inertial sensors for gait analysis in adults with osteoarthritis-a scoping review. Sensors (Switzerland). MDPI AG; 2020. p. 1-24.

14. Zijlstra W, Hof AL. Assessment of spatio-temporal gait parameters from trunk accelerations during human walking. Gait Posture. 2003;18:1-10.

15. Moe-Nilssen R, Helbostad JL. Estimation of gait cycle characteristics by trunk accelerometry. J Biomech Elsevier Ltd. 2004;37:121-6.

16. Moraiti C, Stergiou N, Ristanis S, Georgoulis AD. ACL deficiency affects stride-to-stride variability as measured using nonlinear methodology. Knee Surgery, Sport Traumatol Arthrosc. 2007;15:1406-13.

17. Stergiou N, Decker LM. Human movement variability, nonlinear dynamics, and pathology: Is there a connection? Hum Mov Sci North-Holland. 2011;30:869-88.

18. de Oliveira EA, Andrade AO, Vieira MF. Linear and nonlinear measures of gait variability after anterior cruciate ligament reconstruction. J Electromyogr Kinesiol Elsevier Ltd. 2019;46:21-7.

19. Beck Y, Herman T, Brozgol M, Giladi N, Mirelman A, Hausdorff JM. SPARC: a new approach to quantifying gait smoothness in patients with Parkinson's disease. J Neuroeng Rehabil [Internet]. 2018 [cited 2020 Jan 7];15:49. Available from: https://jneuroengrehab.biomedcentral.com/articles/10.1186/s12984-018-0398-3.

20. Riva F, Toebes MJP, Pijnappels M, Stagni R, van Dieën JH. Estimating fall risk with inertial sensors using gait stability measures that do not require step detection. Gait Posture. 2013;38:170-4.

21. Van Schooten KS, Pijnappels M, Rispens SM, Elders PJM, Lips P, Daffertshofer A, et al. Daily-life gait quality as predictor of falls in older people: A 1-year prospective cohort study. PLoS One. Public Library of Science; 2016;11.

22. Balasubramanian S, Melendez-Calderon A, Roby-Brami A, Burdet E. On the analysis of movement smoothness. J Neuroeng Rehabil [Internet]. 2015 [cited 2019 Dec 12];12:112. Available from: http://www.jneuroengrehab.com/content/12/1/112.

23. Pinto C, Schuch CP, Balbinot G, Salazar AP, Hennig EM, Kleiner AFR, et al. Movement smoothness during a functional mobility task in subjects with Parkinson's disease and freezing of gait - An analysis using inertial measurement units. J Neuroeng Rehabil. BioMed Central Ltd.; 2019;16.

24. Dalton A, Khalil H, Busse M, Rosser A, van Deursen R, ÓLaighin G. Analysis of gait and balance through a single triaxial accelerometer in presymptomatic and symptomatic Huntington's disease. Gait Posture Elsevier. 2013;37:49-54.

25. Pau M, Mandaresu S, Pilloni G, Porta M, Coghe G, Marrosu MG, et al. Smoothness of gait detects early alterations of walking in persons with multiple sclerosis without disability. Gait Posture Elsevier BV. 2017;58:307-9.

26. Pincus SM, Goldberger AL. Physiological time-series analysis: What does regularity quantify? [Internet]. Am. J. Physiol. 1994 [cited 2019 Oct 10]. p. 1643-56. Available from: http://www.ncbi.nlm.nih.gov/pubmed/8184944.

27. Fregly BJ, Besier TF, Lloyd DG, Delp SL, Banks SA, Pandy MG, et al. Grand challenge competition to predict in vivo knee loads [Internet]. J. Orthop. Res. 2012 [cited 2020 Apr 23]. p. 503-13. Available from: http://www.ncbi.nlm.nih.gov/pubmed/22161745.

28. Roetenberg D, Luinge H, Slycke P. Xsens MVN: full 6DOF human motion tracking using miniature inertial sensors. Xsens Technol [Internet]. 2009;8:1-7. Available from: http://www.xsens.com/images/stories/PDF/MVN_white_paper.pdf. 
29. Moe-Nilssen R. A new method for evaluating motor control in gait under real-life environmental conditions. Part 2: Gait analysis. Clin Biomech Elsevier Sci Ltd. 1998;13:328-35.

30. Breniere Y, Do MC. When and how does steady state gait movement induced from upright posture begin? J Biomech. 1986;19:1035-40.

31. Preatoni E, Ferrario M, Donà G, Hamill J, Rodano R. Motor variability in sports: A non-linear analysis of race walking. J Sports Sci. 2010;28:1327-36.

32. Schütte KH, Maas EA, Exadaktylos V, Berckmans D, Venter RE, Vanwanseele B. Wireless tri-axial trunk accelerometry detects deviations in dynamic center of mass motion due to running-induced fatigue. PLoS One. Public Library of Science; 2015;10.

33. Rosenstein MT, Collins JJ, De Luca CJ. A practical method for calculating largest Lyapunov exponents from small data sets. Phys D Nonlinear Phenom. 1993;65:117-34.

34. Bruijn SM, Kate WRT, Ten, Faber GS, Meijer OG, Beek PJ, Dieën JHV. Estimating dynamic gait stability using data from non-aligned inertial sensors. Ann Biomed Eng. 2010;38:2588-93.

35. Bruijn SM, Meijer OG, Beek PJ, Van Dieen JH. Assessing the stability of human locomotion: A review of current measures. J. R. Soc. Interface. Royal Society; 2013.

36. Yentes JM, Hunt N, Schmid KK, Kaipust JP, McGrath D, Stergiou N. The appropriate use of approximate entropy and sample entropy with short data sets. Ann Biomed Eng. 2013;41:349-65.

37. Richman JS, Moorman JR. Physiological time-series analysis using approximate entropy and sample entropy. Am J Physiol Circ Physiol [Internet]. 2000 [cited 2019 Oct 10];278:H2039-49. Available from: https://www.physiology.org/doi/10.1152/ajpheart.2000.278.6.H2039.

38. Melendez-Calderon A, Shirota C, Balasubramanian S. Estimating Movement Smoothness From Inertial Measurement Units. Front Bioeng Biotechnol [Internet]. Frontiers Media S.A.; 2021 [cited 2021 Apr 14];8:558771. Available from: https://www.frontiersin.org/articles/10.3389/fbioe.2020.558771/full.

39. Portney LG, Watkins MP. Foundations of clinical research: applications to practice. Pearson/Prentice Hall; 2009.

40. Virtanen P, Gommers R, Oliphant TE, Haberland M, Reddy T, Cournapeau D, et al. SciPy 1.0: fundamental algorithms for scientific computing in Python. Nat Methods Nature Research. 2020;17:261-72.

41. Astephen Wilson JL, Kobsar D. Osteoarthritis year in review 2020: mechanics. Osteoarthr Cartil [Internet]. 2021;29. Available from: https://doi.org/10.1016/j.joca.2020.12.009.

42. lijima H, Eguchi R, Aoyama T, Takahashi M. Trunk movement asymmetry associated with pain, disability, and quadriceps strength asymmetry in individuals with knee osteoarthritis: a cross-sectional study. Osteoarthr Cartil [Internet]. W.B. Saunders Ltd; 2019 [cited 2020 Oct 22];27:248-56. Available from:

https://pubmed.ncbi.nlm.nih.gov/30445222/.

43. Watelain E, Dujardin F, Babier F, Dubois D, Allard P. Pelvic and lower limb compensatory actions of subjects in an early stage of hip osteoarthritis. Arch Phys Med Rehabil. W.B. Saunders; 2001;82:1705-11.

44. Su JLS, Dingwell JB. Dynamic stability of passive dynamic walking on an irregular surface. J Biomech Eng American Society of Mechanical Engineers Digital Collection. 2007;129:802-10.

45. Tochigi Y, Segal NA, Vaseenon T, Brown TD. Entropy analysis of tri-axial leg acceleration signal waveforms for measurement of decrease of physiological variability in human gait. J Orthop Res. 2012;30:897-904. 
46. Sejdic E, Lowry KA, Bellanca J, Redfern MS, Brach JS. A comprehensive assessment of gait accelerometry signals in time, frequency and time-frequency domains. IEEE Trans Neural Syst Rehabil Eng Institute of Electrical Electronics Engineers Inc. 2014;22:603-12.

47. TenBroek TM, Van Emmerik REA, Hasson CJ, Hamill J. Lyapunov exponent estimation for human gait acceleration signals. J Biomech. 2007;40:210.

\section{Figures}

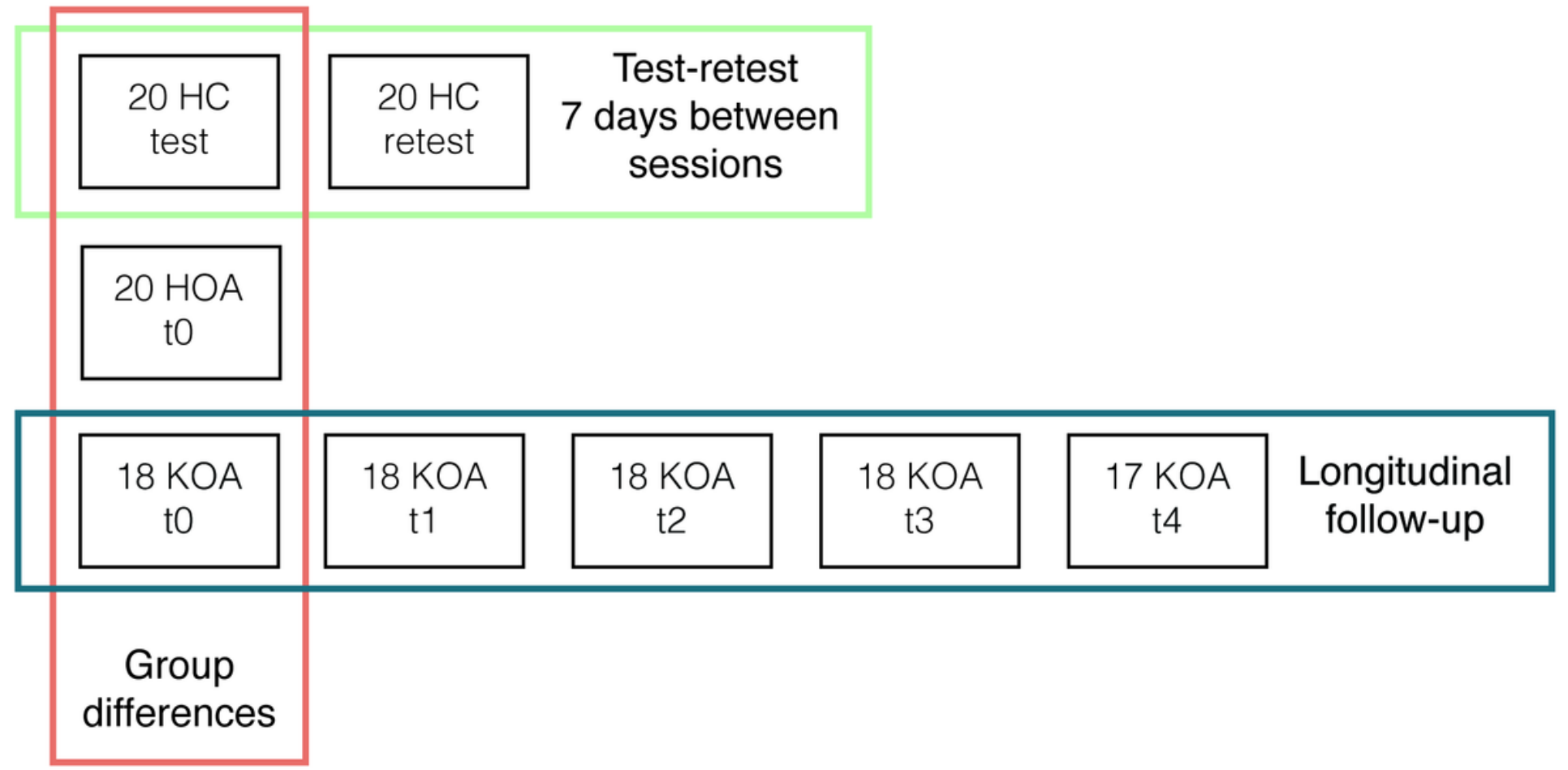

Figure 1

Data collection flow. Legend: Enclosed by the red box is the test-retest reliability data, the yellow box is the longitudinal follow-up data, and the blue box represents the cohort data. $\mathrm{HC}=$ asymptomatic control, $\mathrm{HOA}=$ hip $\mathrm{OA}, \mathrm{KOA}=$ knee OA 
Fig. 2
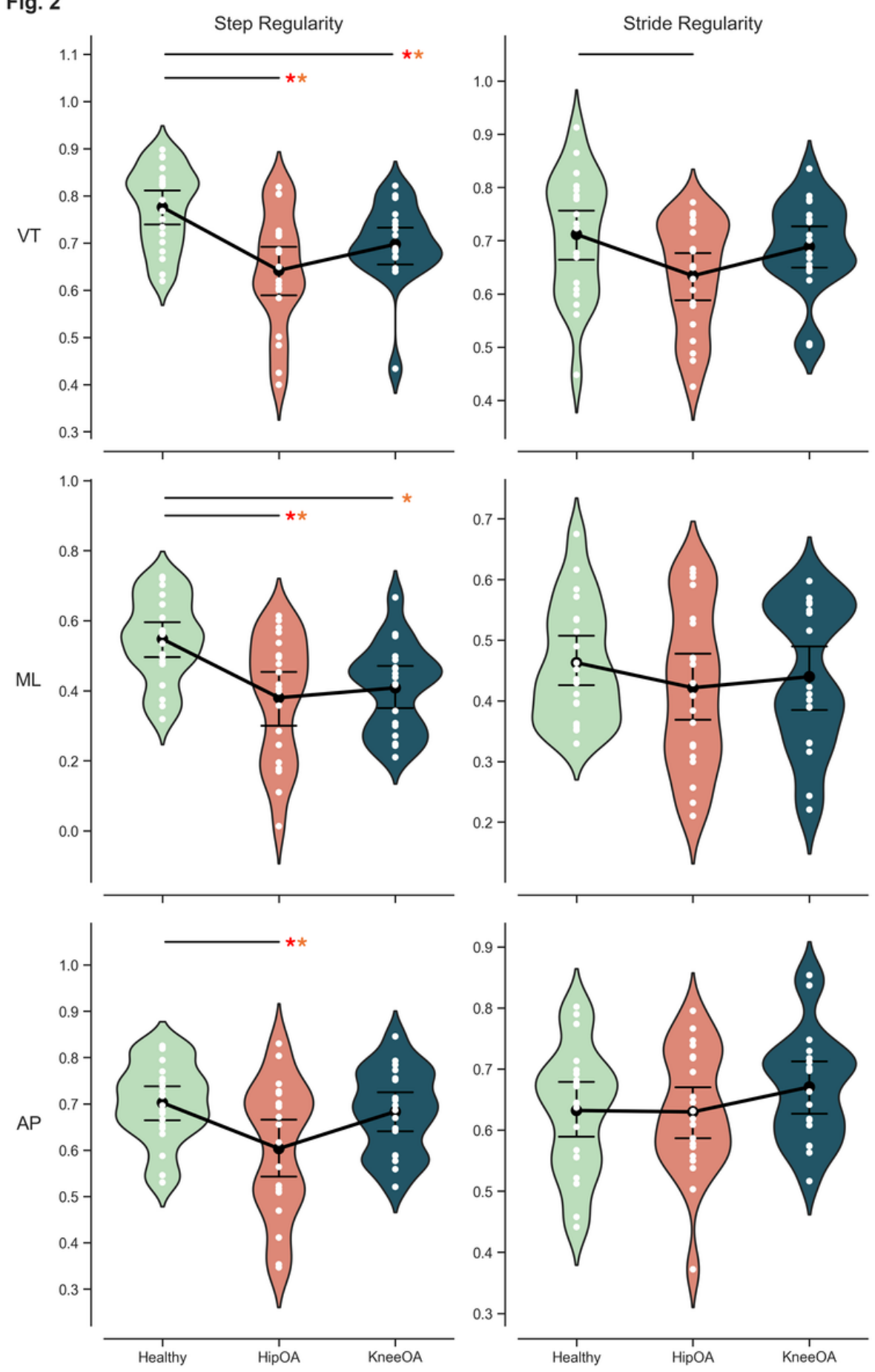

Figure 2

Violin plots of the group differences of symmetry defined by step- and stride regularity. Legend: The white dots represent an individual measurement; the black dot is the mean with the corresponding confidence interval. The violin represents the probability density of the population for each group based on the measured data, (1) healthy in green, (2) hip OA in orange, and (3) knee OA in blue. Black lines above the violins indicate a significant difference between the groups. The red asterisk indicates that the difference exceeds the minimal detectable change (MDC). 
Fig. 3
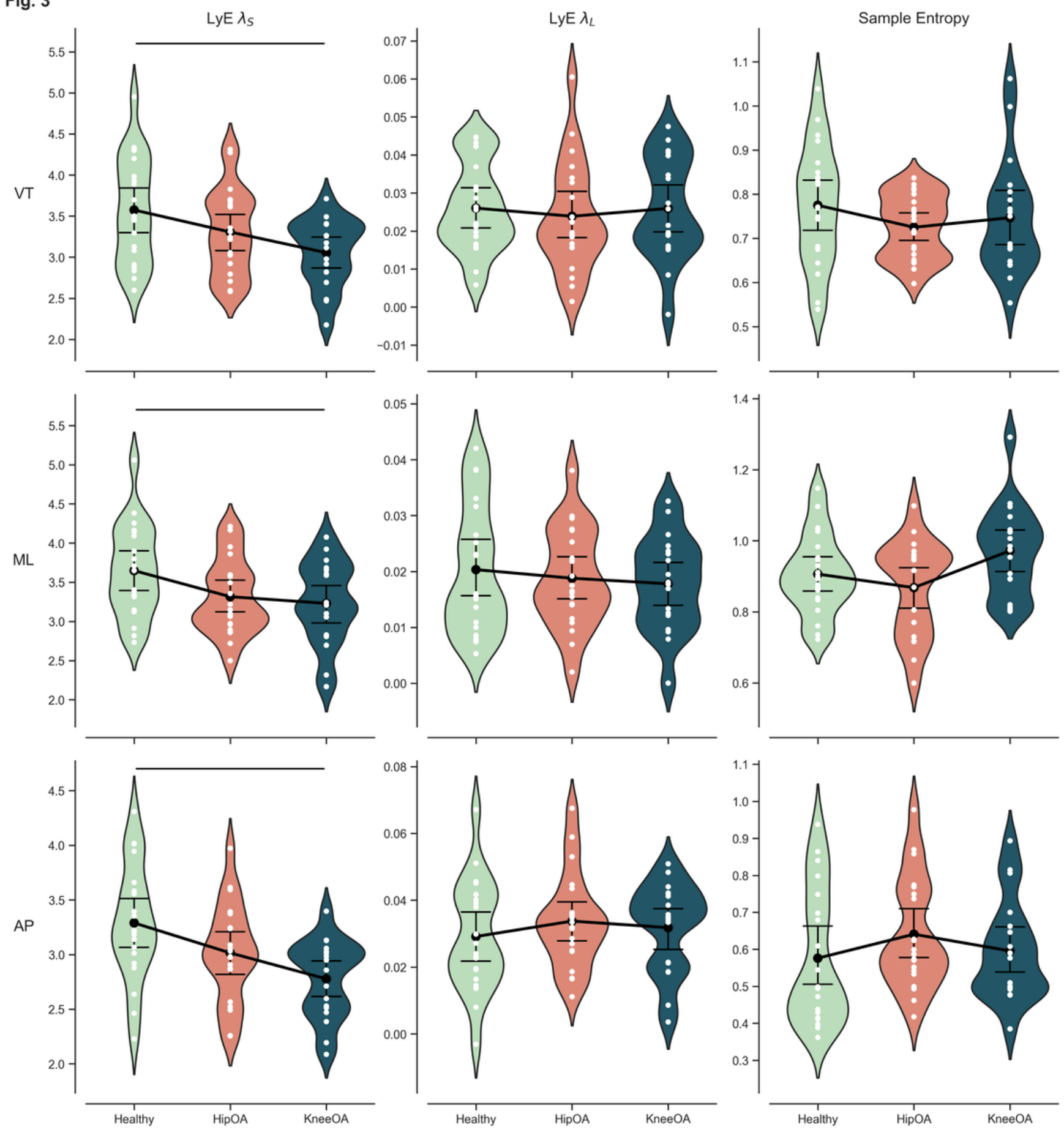

Figure 3

Violin plots of the group differences of stability (Lyapunov Exponent) and complexity (sample entropy). Legend: The white dots represent an individual measurement; the black dot is the mean with the corresponding confidence interval. The violin represents the probability density of the population for each group based on the measured data, (1) healthy in green, (2) hip OA in orange, and (3) knee OA in blue. Black lines above the violins indicate a significant difference between the groups. The red and orange asterisk indicates that the difference exceeds the minimal detectable change (MDC) and $95 \%$ confidence interval, respectively. 
Fig. 4

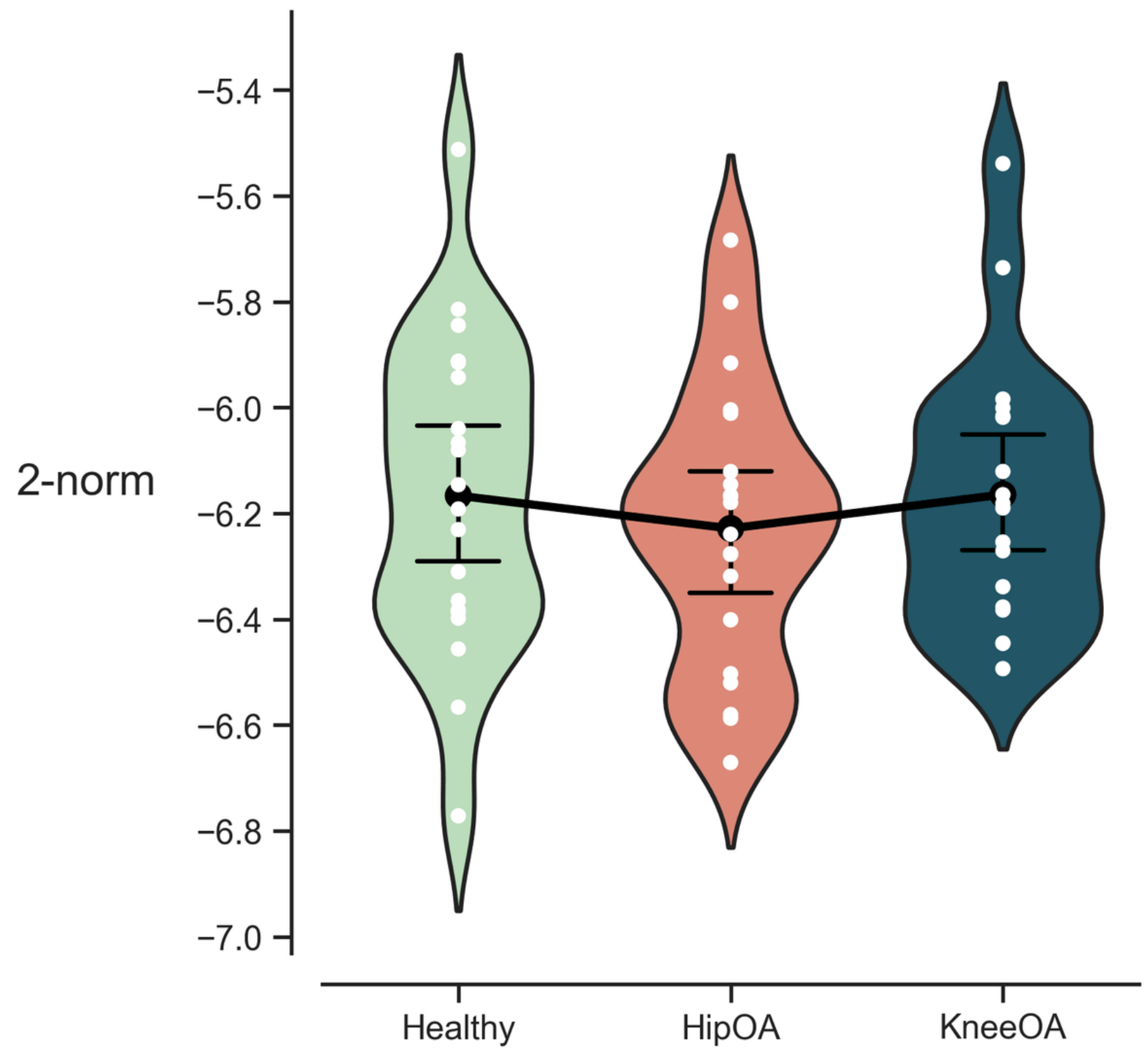

Figure 4

Violin plots of the group differences of smoothness (LDLJ-A) Legend: The white dots represent an individual measurement; the black dot is the mean with the corresponding confidence interval. The violin represents the probability density of the population for each group based on the measured data, (1) healthy in green, (2) hip OA in orange, and (3) knee OA in blue. Black lines above the violins indicate a significant difference between the groups. The red and orange asterisk indicates that the difference exceeds the minimal detectable change (MDC) and $95 \%$ confidence interval, respectively. 
Fig. 5
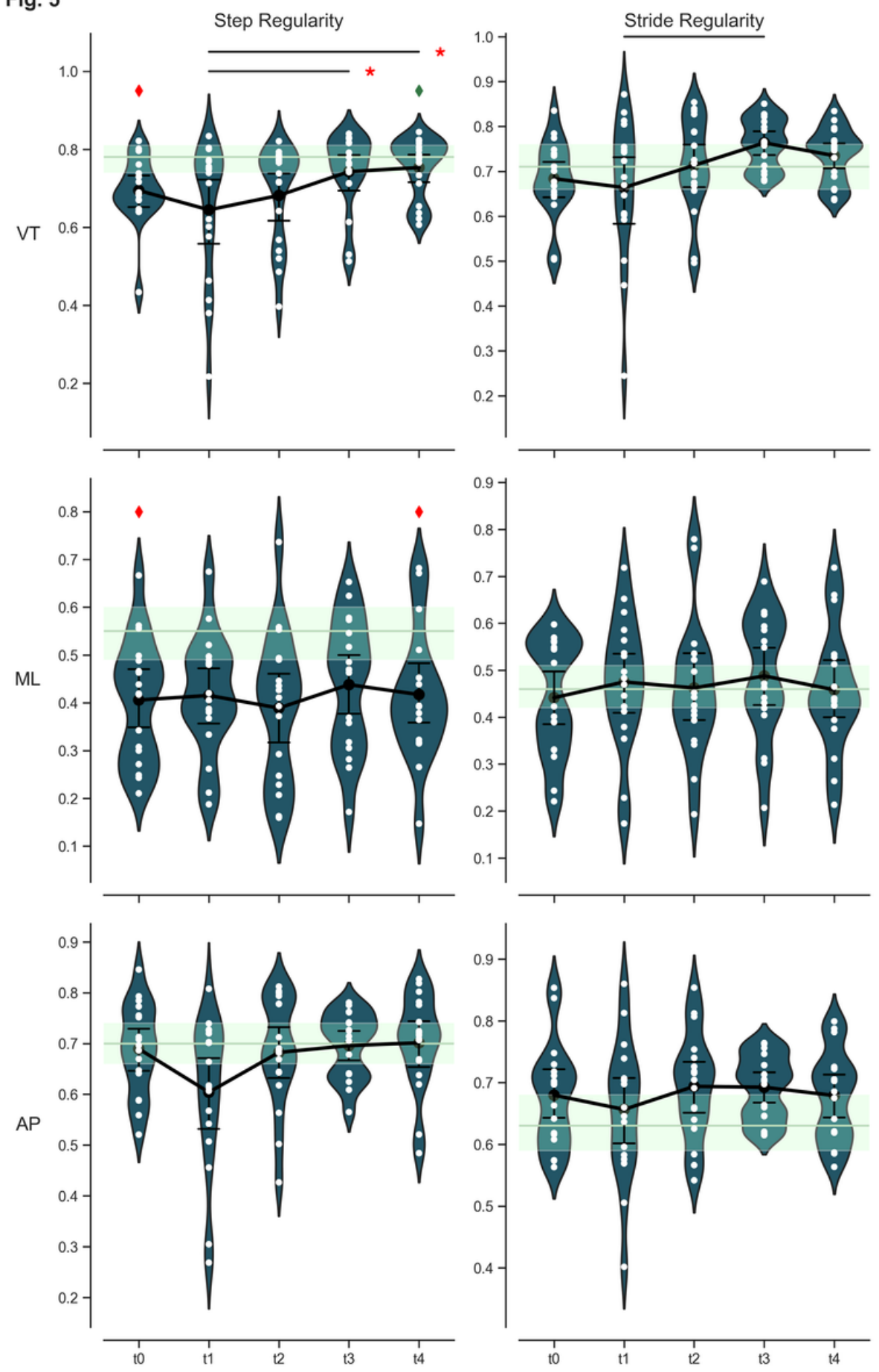

Figure 5

Violin plots of the longitudinal follow-up of gait symmetry post total knee arthroplasty (TKA). Legend: Preoperatively (t0), six weeks, three months, six months and 12 months ( $\mathrm{t} 1-\mathrm{t} 4$, respectively). In the three directions of interest, vertical (VT; row 1), mediolateral (ML; row 2), and anterior-posterior (AP; row 3). The white dots represent an individual measurement; the black dot is the mean with the corresponding confidence interval. The violin represents the probability density of the population for each group based on the measured data. Black lines above the violins indicate a significant difference between the time points. The red asterisk indicates that the difference exceeds the minimal detectable change (MDC). The green shaded bar shows the mean plus confidence interval of the healthy control subjects. A red diamond indicates that that time point was significantly different 
from the healthy controls. Green diamond shows that that difference is no longer significant between 12 months post-op and healthy individuals.

Fig. 6
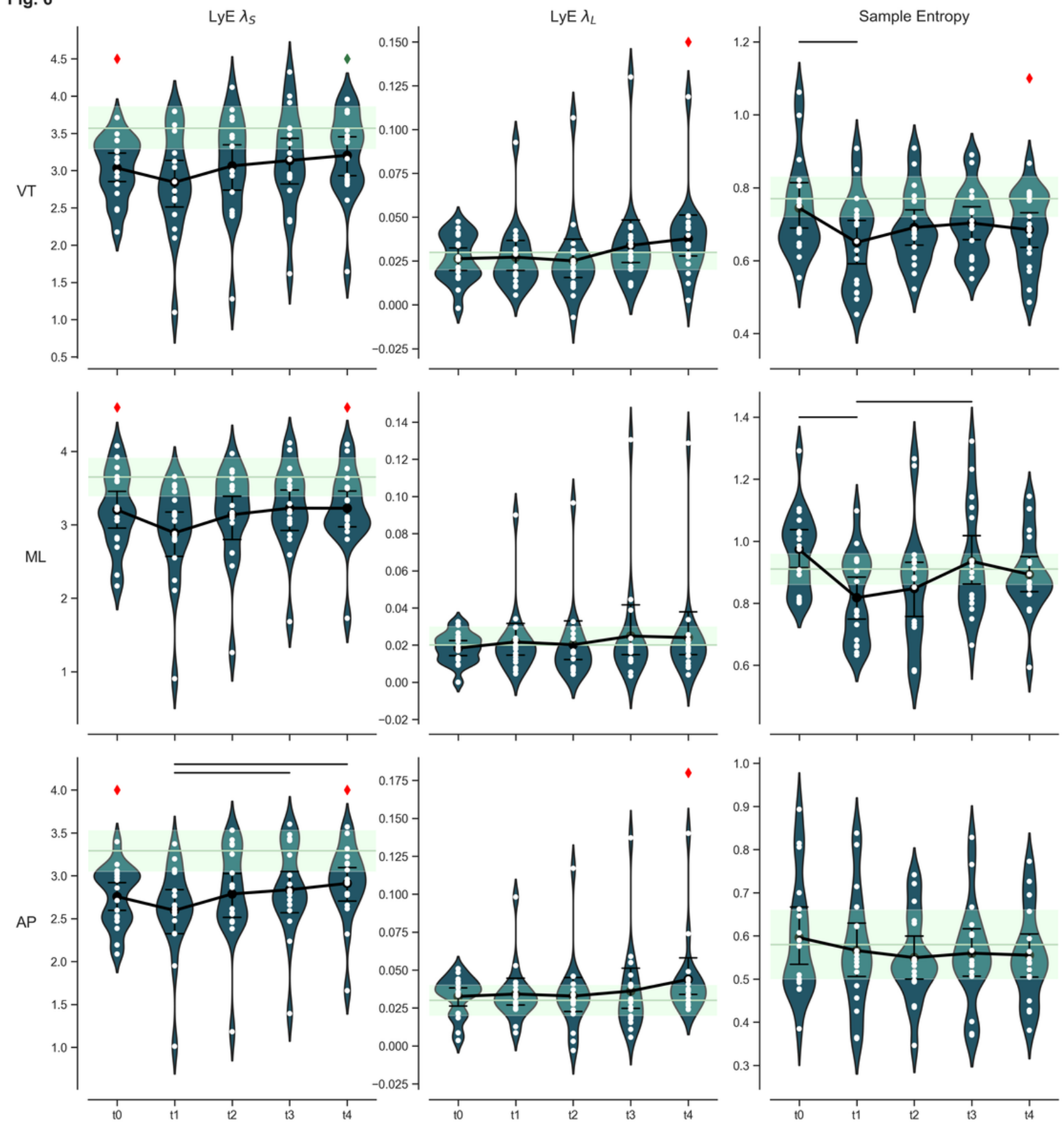

Figure 6

Violin plots of the longitudinal follow-up of stability and smoothness post total knee arthroplasty (TKA). Legend: Pre-operatively (t0), six weeks, three months, six months and 12 months ( $\mathrm{t} 1$ - $\mathrm{t} 4$, respectively). In the three directions of interest, vertical (VT; row 1), mediolateral (ML; row 2), and anterior-posterior (AP; row 3). The white dots represent an individual measurement; the black dot is the mean with the corresponding confidence interval. The violin represents the probability density of the population for each group based on the measured data. Black lines above the violins indicate a significant difference between the time points. The red asterisk indicates that the 
difference exceeds the minimal detectable change (MDC). The green shaded bar shows the mean plus confidence interval of the healthy control subjects. A red diamond indicates that that time point was significantly different from the healthy controls. Green diamond shows that that difference is no longer significant between 12 months post-op and healthy individuals.

Fig. 7

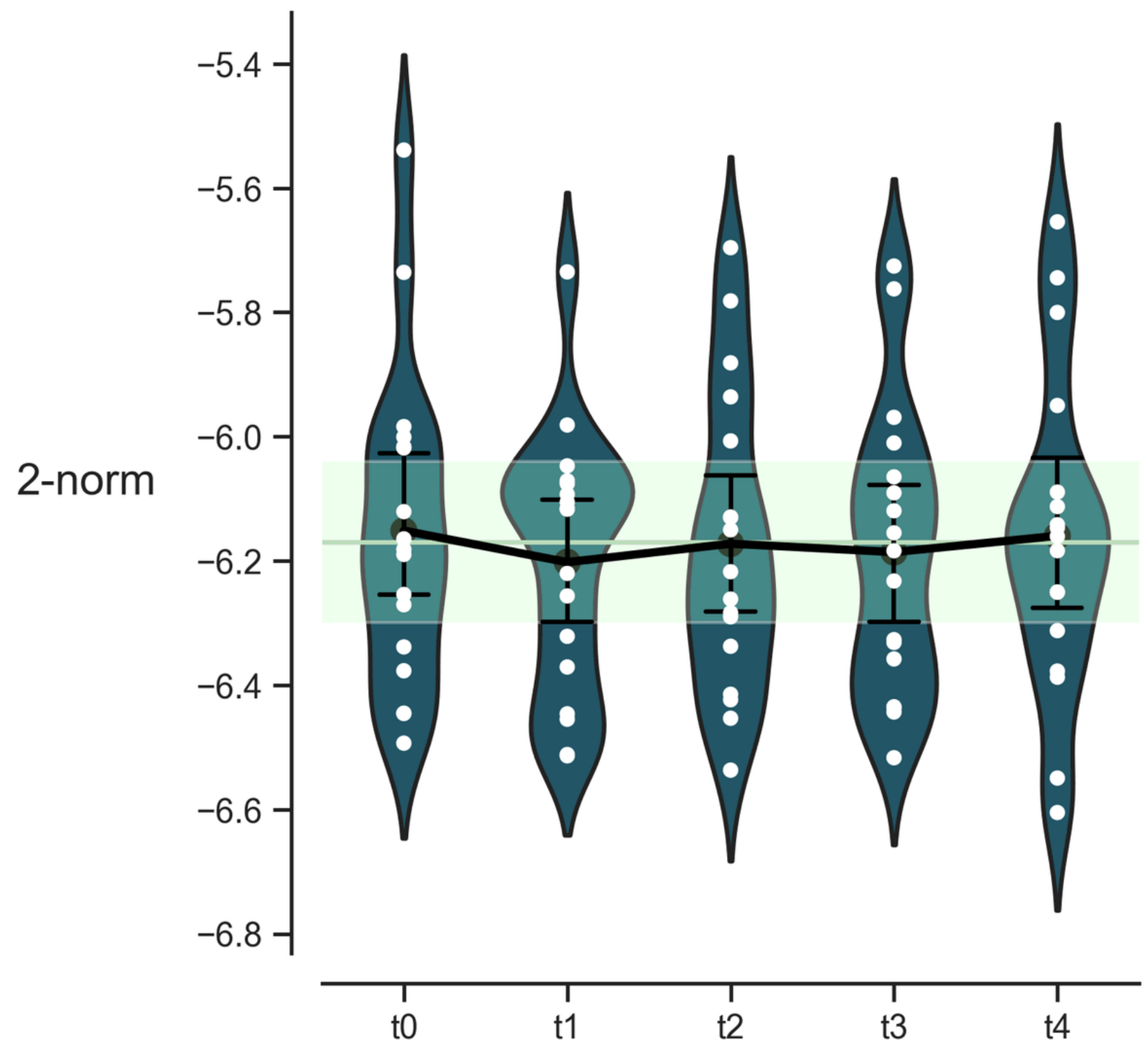

Figure 7

Violin plots of the longitudinal follow-up of gait smoothness post total knee arthroplasty (TKA). Legend: Preoperatively (t0), six weeks, three months, six months and 12 months ( $\mathrm{t} 1-\mathrm{t} 4$, respectively). In the three directions of interest, vertical (VT; row 1), mediolateral (ML; row 2), and anterior-posterior (AP; row 3). The white dots represent an individual measurement; the black dot is the mean with the corresponding confidence interval. The 
violin represents the probability density of the population for each group based on the measured data. Black lines above the violins indicate a significant difference between the time points. The red asterisk indicates that the difference exceeds the minimal detectable change (MDC). The green shaded bar shows the mean plus confidence interval of the healthy control subjects. A red diamond indicates that that time point was significantly different from the healthy controls. Green diamond shows that that difference is no longer significant between 12 months post-op and healthy individuals. 